\title{
Comparative Analysis of SOC Estimation using EECM and NST in Rechargeable $\mathrm{LiCoO}_{2} / \mathrm{LiFePO}_{4} / \mathrm{LiNiMnCoO}_{2} \mathrm{Cells}$
}

\author{
Hyun-jun Lee*, Joung-hu Park* and Jonghoon Kim ${ }^{\dagger}$
}

\begin{abstract}
Lithium rechargeable cells are used in many industrial applications, because they have high energy density and high power density. For an effective use of these lithium cells, it is essential to build a reliable battery management system (BMS). Therefore, the state of charge (SOC) estimation is one of the most important techniques used in the BMS. An appropriate modeling of the battery characteristics and an accurate algorithm to correct the modeling errors in accordance with the simplified model are required for practical SOC estimation. In order to implement these issues, this approach presents the comparative analysis of the SOC estimation performance using equivalent electrical circuit modeling (EECM) and noise suppression technique (NST) in three representative $\mathrm{LiCoO}_{2} / \mathrm{LiFePO}_{4} / \mathrm{LiNiMnCoO}_{2}$ cells extensively applied in electric vehicles (EVs), hybrid electric vehicles (HEVs) and energy storage system (ESS) applications. Depending on the difference between some EECMs according to the number of RC-ladders and NST, the SOC estimation performances based on the extended Kalman filter (EKF) algorithm are compared. Additionally, in order to increase the accuracy of the EECM of the $\mathrm{LiFePO}_{4}$ cell, a minor loop trajectory for proper OCV parameterization is applied to the SOC estimation for the comparison of the performances among the compared to SOC estimation performance.
\end{abstract}

Keywords: SOC estimation, $\mathrm{LiCoO}_{2}$ cell, $\mathrm{LiFePO}_{4}$ cell, $\mathrm{LiNiMnCoO}_{2}$ cell, Equivalent electrical circuit modeling (EECM), Noise suppression technique (NST)

\section{Introduction}

In recent times, the demand for lithium rechargeable cells with high energy density and high discharging voltage, having application in electric-powered applications such as electric vehicles (EVs), hybrid electric vehicles (HEVs) and energy storage system (ESS) has gradually increased [1-11]. Half of the price of these electricpowered applications is related to the battery charge. To ensure that expensive batteries last longer and work efficiently, studies on battery management systems (BMS) are actively being conducted. In particular, when the battery is used in electric-powered application, it has a quick repetition cycle of charging and discharging. Therefore, an accurate state of charge (SOC) estimation is essential for designing a reliable BMS. Because of these reasons, the SOC is considered as one of the most important state variables in BMS.

There are many SOC estimation methods, such as Ahcounting, parameter and heuristic and adaptive methods [12]. The extended Kalman filter (EKF) is one of the adaptive methods [13-18]. The EKF has the advantages of being closed-loop and online as well as the availability of a dynamic SOC estimation error range. Thus, an application

$\dagger$ Corresponding Author: Dept. of Electrical Engineering, Chungnam

National University, Korea. (whdgns0422@cnu.ac.kr)

* Dept. of Electrical Engineering, Soongsil University, Korea.

(jun2u@naver.com, wait4u@ssu.ac.kr)

Received: November 12, 2015 ; Accepted: June 20, 2016 of a simplified nonlinear electrical equivalent circuit model (EECM) is possible. The correctness of the EECM determines the accuracy of the model-based SOC estimation. The number of RC-ladders plays a significant role in determining the performance of the SOC estimation about the dynamic characteristics of the battery. The RCladder showing the diffusion of the battery and it consists of a series combination of several RC-ladders. However, if the modeling of the diffusion region consists of an infinite number of RC-ladders, the configuration of the algorithm and calculation is significantly difficult and complex. Thus, the EECM is simplified as a combination of the first or second order RC-ladder only as it has the greatest resistance value. In the EKF algorithm, the internal state of the battery predicted by the state equation is modified by a measurement equation. However, the internal state can be guided in wrong directions if the measurements are inaccurate or includes a large noise due to system factors. Model error caused by the use of a simplified EECM is also included in the noise. The method used to compensate for the noise caused by model errors is called noise suppression technique (NST) $[19,20]$. In the EKF, the Kalman gain determines the effect of the measurement equation. When noise is generated by the model errors, the NST decreases the Kalman gain to reduce the effect of the measurement equation.

In this overall perspective, this approach implements the comparative analysis of the SOC estimation that absolutely 
depends on the EECM structure and the NST in commercial representative lithium cobalt oxide $\left(\mathrm{LiCoO}_{2} ; \mathrm{LCO}\right)$, lithium iron phosphate $\left(\mathrm{LiFePO}_{4} ; \mathrm{LFP}\right)$ and lithium nickel manganese cobalt oxide ( $\mathrm{LiNiMnCoO}_{2}$; NMC) popularly in EVs, HEVs and ESS applications. Basically, for three cells, it is compared how the accuracy of the SOC estimation changed according to the number of RC-ladder and NST. The most important thing to be considered in this approach is that the $\mathrm{LiFePO}_{4}$ cell has unusual open-circuit voltage $(\mathrm{OCV})$ characteristics caused by the hysteresis effect. Therefore, it is so difficult to have an accurate SOC estimation using a conventional OCV, namely major loop [20-23]. The accuracy of the EECM can be more increased by developing some minor loops in the conventional charging/discharging OCV curves (major loop). For this critical reason, this approach further investigated the comparative analysis of accuracy of SOC estimation with and without the minor loop tractor of the OCV. In this overall perspective, the purpose of the proposed approach is to provide important solutions of the EECM structure (RC-ladder number and hysteresis effect) and the NST for achieving high accuracy SOC performance of three representative $\mathrm{LiCoO}_{2}, \mathrm{LiFePO}_{4}$ and $\mathrm{LiNiMnCoO}_{2}$ cells.

\section{Experimental Apparatus}

Fig. 1 shows an experimental apparatus for testing the proposed approach. For the experiment, one $\mathrm{LiCoO}_{2}$

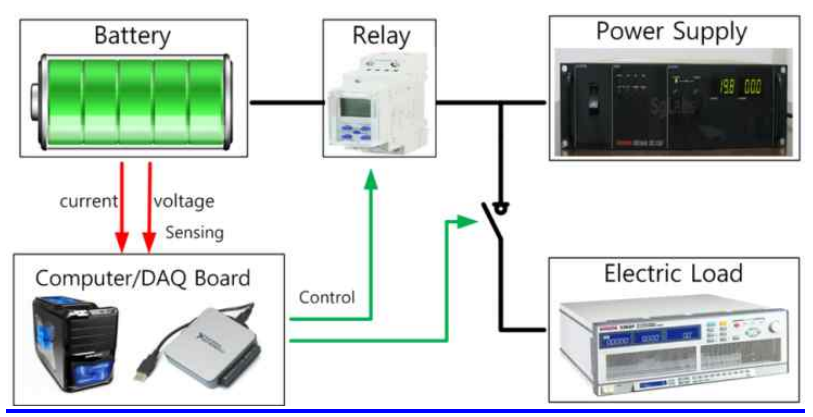

Fig. 1. Experimental apparatus

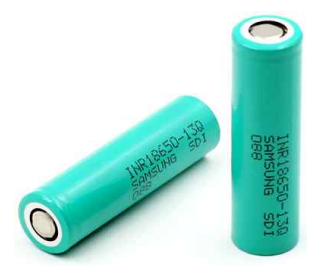

(a)

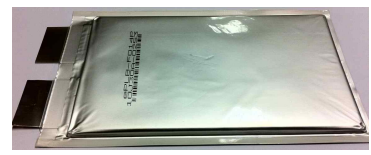

(b)

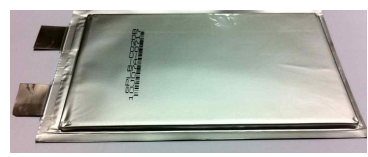

(c)
Fig. 2. Experimental cells considered in this approach: (a) $\mathrm{LiCoO}_{2}$ cell (1.3Ah, Samsung SDI); (b) $\mathrm{LiFePO}_{4}$ cell(14Ah, EIG); (c) $\mathrm{LiNiMnCoO}_{2}$ cell(20Ah, EIG) cylindrical cell and one $\mathrm{LiFePO}_{4}$ and one $\mathrm{LiNiMnCoO}_{2}$ pouch type cells respectively having high capacity of 1.3Ah, 14Ah and 20Ah are used (see Fig. 2). The experimental apparatus is configured with a power supply for battery charging (Sorenson DCS55-55) and an electric load for discharging (Prodigit 3362F). When the relay is on and off, the charging and discharging programs in the computer control the battery's current and voltage. Further, the experimental voltage and current data are collected by the DAQ board (NI USB-6000) and stored in the computer. With collected data, the SOC estimation is conducted using MATLAB/Simulink.

\section{Fundamental Review of Three Key Factors for SOC Estimation}

\subsection{Review of the EECM}

Irrespective of kind of battery, the EECM of the battery consists of one series resistance $\mathrm{R}_{\mathrm{i}}$, one RC-ladder and the OCV, as shown in Figs. 3(a). $R_{i}$ represents the internal resistance and the charge transfer phenomenon of the battery. The RC-ladder is composed of a parallel combination of $\mathrm{R}_{\text {diff }}$ and $\mathrm{C}_{\text {diff }}$, which represents the diffusion region, made up of an infinite number of pairs. However, if the region is modeled with an infinite number of RC-ladders, theoretical implementation of the designing a model and algorithm calculation is very difficult and the derivation procedure is complex. In addition, a high-cost digital processor is needed to build a real application. As the ladder count $n$ increases, the value of resistance $\mathrm{R}_{\text {diff, }}$, which is inversely

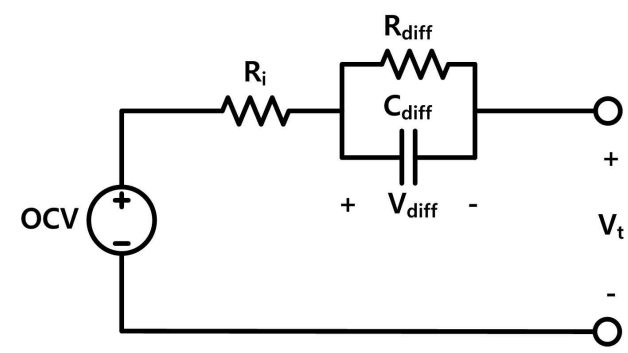

(a)

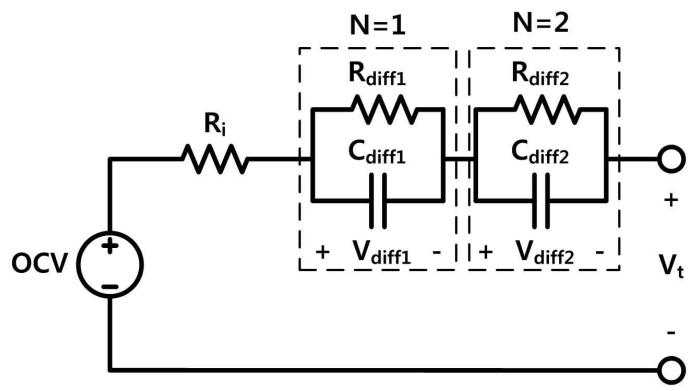

(b)

Fig. 3. Equivalent electrical circuit model (EECM) of the $\mathrm{LiCoO}_{2}$ cell and $\mathrm{LiNiMnCoO}_{2}$ cell: (a) Simplified EECM of the $\mathrm{LiCoO}_{2}$ and $\mathrm{LiNiMnCoO}$ cells; (b) 
proportional to $n^{2}$ is decreased as shown in Figs. 3(b). In accordance with the decrease in $\mathrm{R}_{\text {diff }}$, the time constant $\tau$ of the RC-ladder and the effect of the capacitance also decrease. As a result, the diffusion region can be simplified as a first or a second-order RC-ladder and it has the largest capacitance value. The number of this RC-ladder is directly connected with the accuracy of the model-based SOC estimation performance. Charging/discharging OCV curves of the $\mathrm{LiCoO}_{2}$ and $\mathrm{LiNiMnCoO}$ cells in the entire SOC range for except for SOC $0 \%$ and 100\% are shown in Figs. 4 and 5 , respectively. It can be known that there is little difference between charging OCV and discharging OCV. In addition, this OCV is approximately linear. Therefore, it is possible to consider a unique representative OCV for SOC estimation, as shown in Fig. 3(a) and (b).

Fig. 6 represents that the EECM of the $\mathrm{LiFePO}_{4}$ is quite similar with those of the $\mathrm{LiCoO}_{2}$ and $\mathrm{LiNiMnCoO}_{2}$ cells. In contrast to the $\mathrm{LiCoO}_{2}$ cell and $\mathrm{LiNiMnCoO}$ cell, the most important thing to be considered in the $\mathrm{LiFePO}_{4}$ cell is that there is inevitable hysteresis effect [21], known as the major loop. Both the charging and discharging OCV curves of the $\mathrm{LiFePO}_{4}$ cell are very flat and they have a significant difference caused by the hysteresis phenomenon (see Fig. 7). Therefore, as the OCV part specifically shown in Fig. 6, the OCV modeling of $\mathrm{LiFePO}_{4}$ cell is inevitably divided into the parts of charging and discharging $\mathrm{OCV}$, unlike the modeling of the $\mathrm{LiCoO}_{2}$ and $\mathrm{LiNiMnCoO}_{2}$ cells. A minor loop can be further applied to describe the hysteresis

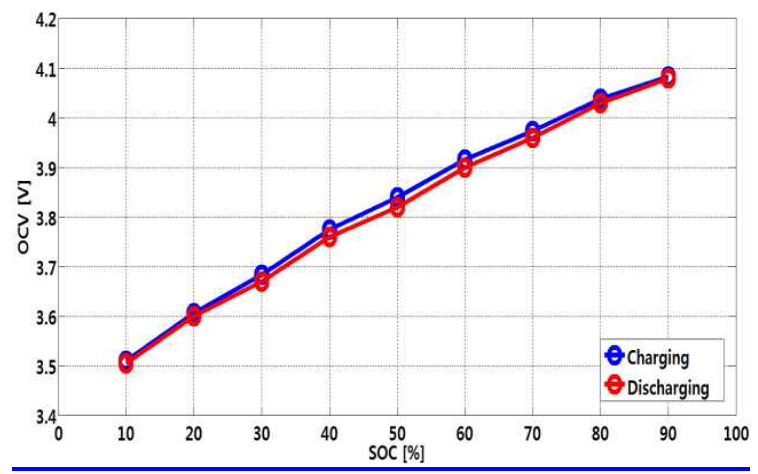

Fig. 4. Charging/discharging OCV curves of the $\mathrm{LiCoO}_{2}$ cell (1.3Ah).

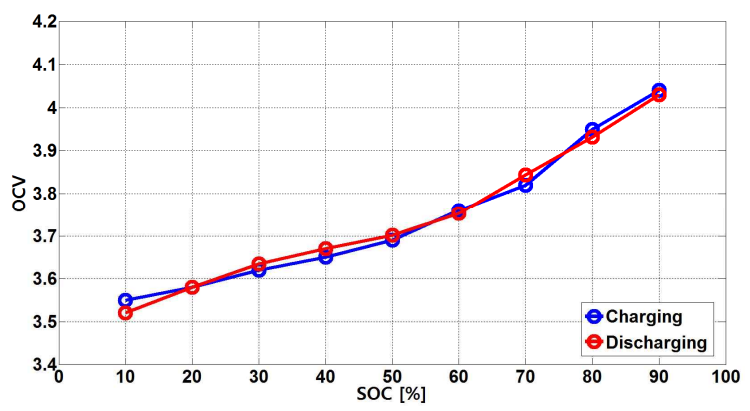

Fig. 5. Charging/discharging OCV curves of the $\mathrm{LiNiMnCoO}_{2}$ cell (20Ah) phenomenon, as shown in Fig. 8. Minor loop hysteresis is designed by a first-order function.

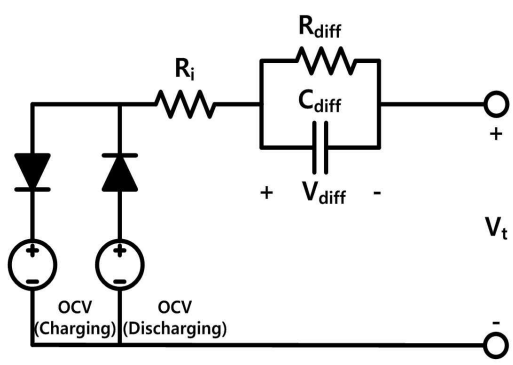

(a)

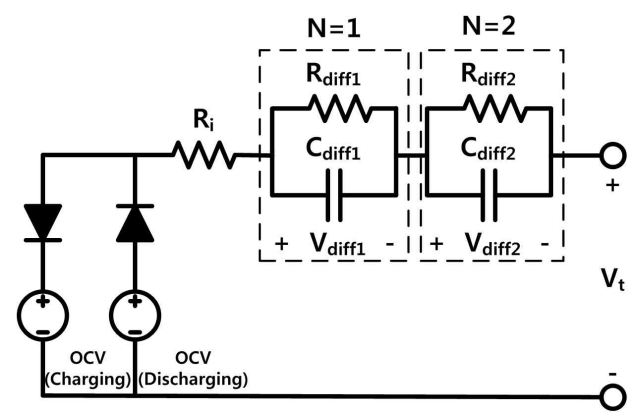

(b)

Fig. 6. Equivalent electrical circuit model (EECM) of the $\mathrm{LiFePO}_{4}$ cell: (a) Simplified EECM of the $\mathrm{LiFePO}_{4}$ cell; (b) EECM with two RC-ladders of the $\mathrm{LiFePO}_{4}$ cells

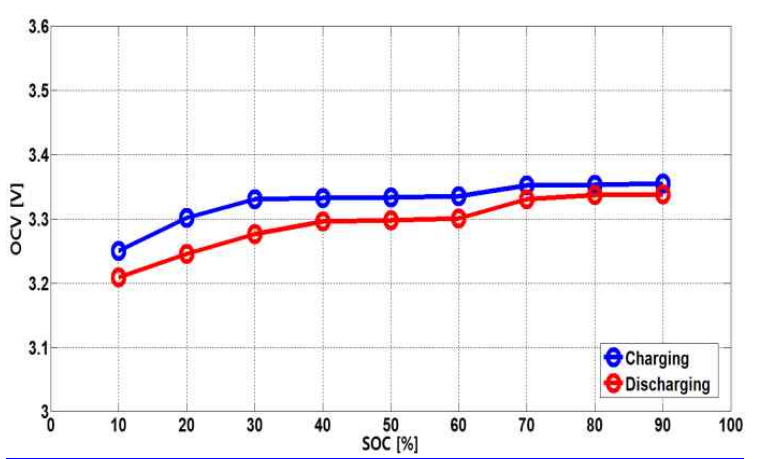

Fig. 7. Charging/discharging OCV curves of the $\mathrm{LiFePO}_{4}$ cell (14Ah)

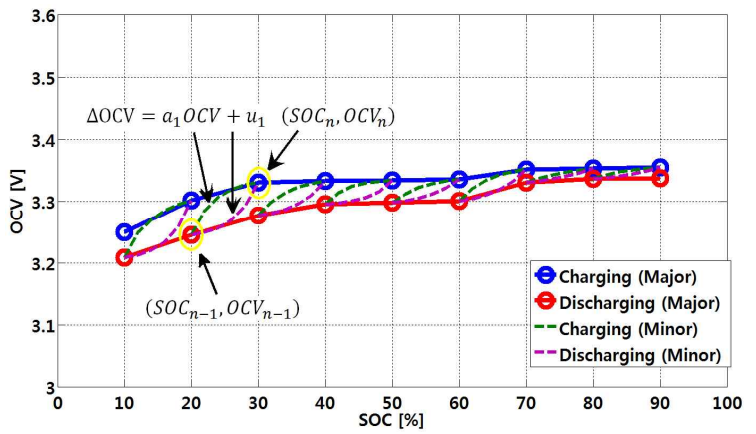

Fig. 8. Major and minor loops of the $\mathrm{LiFePO}_{4}$ cell (14Ah) 


$$
\begin{aligned}
& \operatorname{OCV}_{n}\left(\operatorname{SOC}_{n}\right)=\mathrm{OCV}_{n-1}+\int_{\operatorname{SOC}_{n-1}}^{\operatorname{SOC}_{n}} e^{a_{1}\left(\operatorname{SOC}_{n}-t\right)} \cdot u_{1} d t \\
& \operatorname{OCV}_{n}\left(\operatorname{SOC}_{n}\right)=\mathrm{OCV}_{n-1}-\int_{\operatorname{SOC}_{n-1}}^{\operatorname{SOC}_{n}} e^{a_{1}\left(\operatorname{SOC}_{n}-t\right)} \cdot u_{1} d t
\end{aligned}
$$

with a slope $a_{1}$ and y-intercept $u_{1}$ in the major loop area, between discharging $\mathrm{OCV}_{n-1}$ and charging $\mathrm{OCV}_{n}$ [21]. Each of the design equations is a first-order differential equation form representing the exponential function. Eqs. (1) and (2) are examples of the minor loop equations in the charging and discharging directions, respectively. However, in applications such as EVs or renewable energy systems, charging and discharging OCVs often iterate in a short period, as the battery cell repeats the charging and discharging cycles in a small SOC range in real application, such as EVs and HEVs. Then, multiple minor hysteresis loops by SOC $10 \%$ interval more effective, as illustrated in Fig. 8 [22]. The values of $a_{1}$ and $u_{1}$ can be flexibly changed according to the accuracy of the SOC estimation.

\subsection{Review of the EKF algorithm}

Because the EKF algorithm has more high accuracy and strong reliability against noise, it is possible to consider the simplified EECM. According to the optimal state recursive estimation in the EKF, two major equations for expressing the dynamic and measurement models has been used. First of all, for expression of the dynamic model, the state equation is considered. The state equation has the process noise of the state filter with assumption of zero-mean, independency, and Gaussian noise with a covariance matrix. When EKF initialization is performed, the states are preferentially set to be best guess based on the prior information $(k=0)$. At each time step with $(k=\{1, \cdots, \infty\})$, the state equation continuously implements the time-update. Then, updated state information is simultaneously applied to the measurement model, namely measurement equation. From this rule, it can be sufficiently expected to adapt the state using the estimation error from comparison between the real output and the estimated output. As a result, it is possible to calculate the posteriori state estimate from correcting the priori state with the estimation error multiplied by gain factor.

Eqs. (3)-(4) respectively shows the state equation of the EKF when the number of RC-ladders is one or two. As expressed in (3)-(4), there are two states of SOC and $V_{\text {diff }}$ properly estimated under consideration of terminal voltage and input current $i_{k}$

$$
\left[\begin{array}{l}
\mathrm{SOC}_{k} \\
\mathrm{~V}_{\mathrm{diff}, k}
\end{array}\right]=\left[\begin{array}{cc}
1 & 0 \\
0 & 1-\frac{\Delta t}{\mathrm{R}_{\mathrm{diff}} \mathrm{C}_{\mathrm{diff}}}
\end{array}\right]\left[\begin{array}{l}
\mathrm{SOC}_{k-1} \\
\mathrm{~V}_{\mathrm{diff}, k-1}
\end{array}\right]+\left[\begin{array}{c}
-\frac{\Delta t}{\mathrm{C}_{n}} \\
\frac{\Delta t}{\mathrm{C}_{\mathrm{diff}}}
\end{array}\right] i_{k-1}
$$

$$
\begin{aligned}
& {\left[\begin{array}{c}
\mathrm{SOC}_{k} \\
\mathrm{~V}_{\text {diff } 1, k} \\
\mathrm{~V}_{\text {diff } 2, k}
\end{array}\right]=\left[\begin{array}{ccc}
1 & 0 & 0 \\
0 & 1-\frac{\Delta t}{\mathrm{R}_{\text {diff }} \mathrm{C}_{\text {diff }}} & 0 \\
0 & 0 & 1-\frac{4 \Delta t}{\mathrm{R}_{\text {diff }} \mathrm{C}_{\text {diff }}}
\end{array}\right]\left[\begin{array}{c}
\mathrm{SOC}_{k-1} \\
\mathrm{~V}_{\text {diff } 1, k-1} \\
\mathrm{~V}_{\text {diff } 2, k-1}
\end{array}\right]} \\
& +\left[\begin{array}{c}
-\frac{\Delta t}{\mathrm{C}_{n}} \\
\frac{2 \Delta t}{\mathrm{C}_{\mathrm{diff}}} \\
\frac{2 \Delta t}{\mathrm{C}_{\mathrm{diff}}}
\end{array}\right] i_{k-1} \\
& \mathrm{~V}_{k}=h_{k}\left(\mathrm{OCV}, \mathrm{V}_{\text {diff }}\right)-\mathrm{R}_{i} i_{k}=\mathrm{OCV}-\mathrm{V}_{\text {diff }}-\mathrm{R}_{i} i_{k} \\
& \mathrm{~V}_{k}=h_{k}\left(\mathrm{OCV}, \mathrm{V}_{\text {diff } 1}, \mathrm{~V}_{\text {diff } 2}\right)-\mathrm{R}_{i} i_{k} \\
& =\mathrm{OCV}-\mathrm{V}_{\text {diff } 1}-\mathrm{V}_{\text {diff } 2}-\mathrm{R}_{i} i_{k}
\end{aligned}
$$

Eqs. (5)-(6) respectively present the measurement equation of the EKF with one or two RC-ladders. From incorporated states at each time step $k$, it can be absolutely checked that two states of SOC and $\mathrm{V}_{\text {diff }}$ updated in the state equation are sequentially applied to the measurement equation expressed by a nonlinear function at the same time. From Eqs. (5)-(6), the performance on the SOC estimation is intimately connected with the OCV.

\subsection{Review of the NST}

Estimation errors are sometimes caused in the process of EECM simplification and while implementing variations in the parameters of the EECM. The reduced-order model includes slow dynamics of the simplified EECM, but excludes the fast dynamic responses, which causes the model error and inevitably leads to an inaccurate estimation result. To overcome the simplified EECM error, the measurement noise models and data rejection techniques are absolutely required. Given in the covariance matrix of the state estimation uncertainty $P_{k}$ and the measurement sensitivity matrix $H_{k}$, the measurement noise model is properly controlled by the measurement noise covariance, $R_{k}$, which is an important value in determining the gain $\left(K_{k}\right)$ of the Kalman filter, as expressed in Eqs. (7) and (8). The value of $K_{k}$ is inversely related to the value of $R_{k}$. Three noise model and data rejection techniques for each cell are presented in Tables 1 through 3. The measurement noise model defined by the battery current is applied to avoid the voltage error when a large charging/discharging current is imposed. The measurement noise model of dynamic response of the RC-ladder is applied to the dynamic characteristics of the RC-ladder with an imposed step current. The data rejection operates when the EECM error abruptly grows higher. So, this NST including the measurement noise model and the 
Table 1. Noise model and data rejection in the EKF of the $\mathrm{LiCoO}_{2}$ cell

\begin{tabular}{l}
\hline Measurement noise model by battery current \\
\hline$R_{k+1}=R_{k}$, reliable current $(|i|<4 \mathrm{~A})$ \\
$R_{k+1}=R_{k}\left[1+G_{i}(|i|-4 \mathrm{~A})\right]$, unreliable current $(|i|>4 \mathrm{~A}) \quad G_{i}=2 \mathrm{~A}^{-1}$ \\
\hline Measurement noise model by dynamic of RC-ladder \\
\hline$R_{k+1}=R_{k}\left[1+G_{\text {step }}(\right.$ step_time $\left.)\right] \quad G_{\text {step }}=0.1 \mathrm{sec}^{-1}$ \\
\hline Data rejection technique \\
\hline$R_{k}=\infty$, reject time $(\Delta I>4 \mathrm{~A}) \quad$ reject time $=10 \mathrm{~ms}$ \\
\hline
\end{tabular}

Table 2. Noise model and data rejection in the EKF of the $\mathrm{LiFePO}_{4}$ cell

\begin{tabular}{l}
\hline Measurement noise model by battery current \\
\hline$R_{k+1}=R_{k}$, reliable current $(|i|<10 \mathrm{~A})$ \\
$R_{k+1}=R_{k}\left[1+G_{i}(|i|-10 \mathrm{~A})\right]$, unreliable current $(|i|>10 \mathrm{~A}) \quad G_{i}=4 \mathrm{~A}^{-1}$ \\
\hline Measurement noise model by dynamic of RC-ladder \\
\hline$R_{k+1}=R_{k}\left[1+G_{\text {step }}(\right.$ step_time $\left.)\right] \quad G_{\text {step }}=0.1 \mathrm{sec}^{-1}$ \\
\hline Data rejection technique \\
\hline$R_{k}=\infty$, reject time $(\Delta I>10 \mathrm{~A}) \quad$ reject time $=10 \mathrm{~ms}$ \\
\hline
\end{tabular}

Table 3. Noise model and data rejection in the EKF of the $\mathrm{LiNiMnCoO}_{2}$ cell

\begin{tabular}{l}
\hline Measurement noise model by battery current \\
\hline$R_{k+1}=R_{k}$, reliable current $(|i|<6 \mathrm{~A})$ \\
$R_{k+1}=R_{k}\left[1+G_{i}(|i|-6 \mathrm{~A})\right]$, unreliable current $(|i|>6 \mathrm{~A}) \quad G_{i}=3 \mathrm{~A}^{-1}$ \\
\hline Measurement noise model by dynamic of $\mathrm{RC}$-ladder \\
\hline$R_{k+1}=R_{k}\left[1+G_{\text {step }}(\right.$ step_time $\left.)\right] \quad G_{\text {step }}=0.1 \mathrm{sec}^{-1}$ \\
\hline Data rejection technique \\
\hline$R_{k}=\infty$, reject time $(\Delta I>6 \mathrm{~A}) \quad$ reject time $=10 \mathrm{~ms}$ \\
\hline
\end{tabular}

data rejection technique is linked closely with SOC performance.

\section{Comparison of Three Representative Cells}

\subsection{EECM and SOC estimation of the $\mathrm{LiCoO}_{2}$ cell}

\subsubsection{Related to the number of RC-ladder}

When the scaled-down HEV charging/discharging current profile is applied to the cell, Fig. 9 shows the comparison of SOC estimation related to the number of RC-ladders in the $\mathrm{LiCoO}_{2}$ cell, namely parametrization. The accuracy of SOC estimation of the two RC-ladders in the EECM is higher than when it is one and lower as compared with the Ah-counting SOC estimation. Fig. 10 illustrates the difference of the SOC estimation between Ah-counting and result employing one RC-ladder model and two RC-ladders in the EECM. Although the difference between the average value of the SOC estimation error is not large, as shown in Fig. 10(b), it can be confirmed that the two RC-ladder EECM is more flexible to address the repeated charging and discharging, as shown in Fig. 10(a).

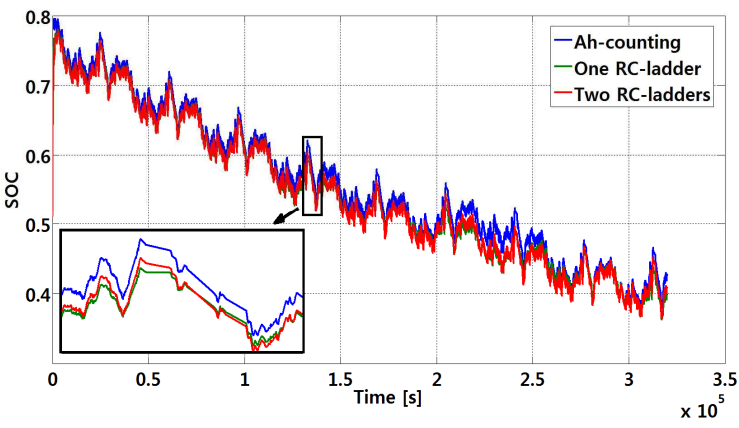

Fig. 9. Comparison of SOC estimation results related to the number of RC-ladder of the $\mathrm{LiCoO}_{2}$ cell

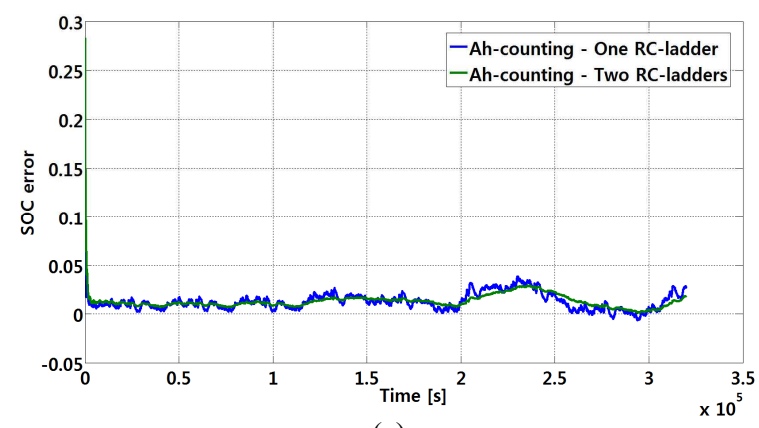

(a)

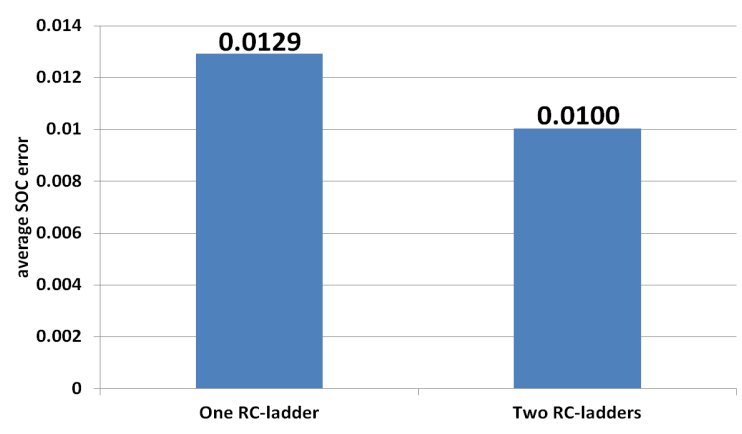

(b)

Fig. 10. Comparison of SOC estimation errors related to the number of RC-ladder of the $\mathrm{LiCoO}_{2}$ cell: (a) Comparison of SOC estimation errors; (b) Comparison of average SOC estimation errors

\subsubsection{Related to the NST}

Under consideration of one and two RC-ladders, Fig. 11 shows the comparison of the SOC estimation graph related to the NST of the $\mathrm{LiCoO}_{2}$ cell. The result of the SOC estimation is more exact when the NST is used. In addition, it can be seen that the effect of the NST is greater than that of the number of RC-ladder, as shown in Fig. 12. Two measurement noise models and the data rejection technique were considered as the NST. The $\mathrm{LiCoO}_{2}$ cell is sensitive to external physical factors like changes in the temperature and current. Therefore, the NST is more effective in $\mathrm{LiCoO}_{2}$ cell SOC estimation than the number of RC-ladder. 


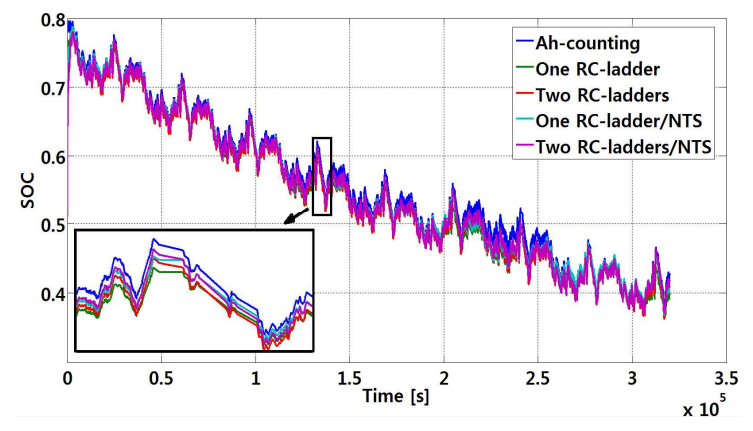

Fig. 11. Comparison of SOC estimation results related to the NST of the $\mathrm{LiCoO}_{2}$ cell

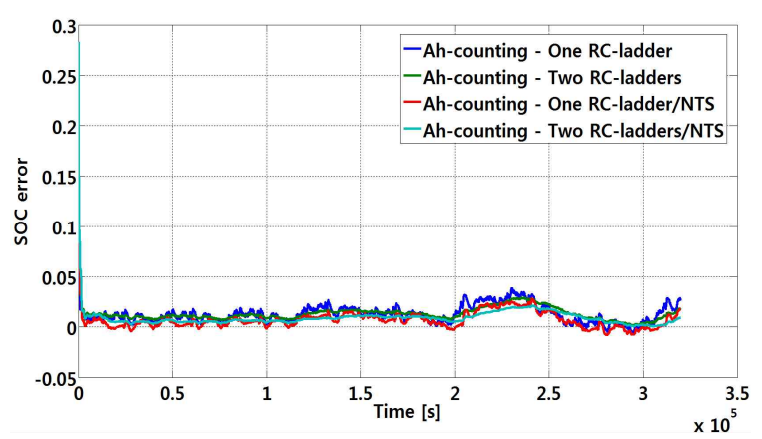

(a)

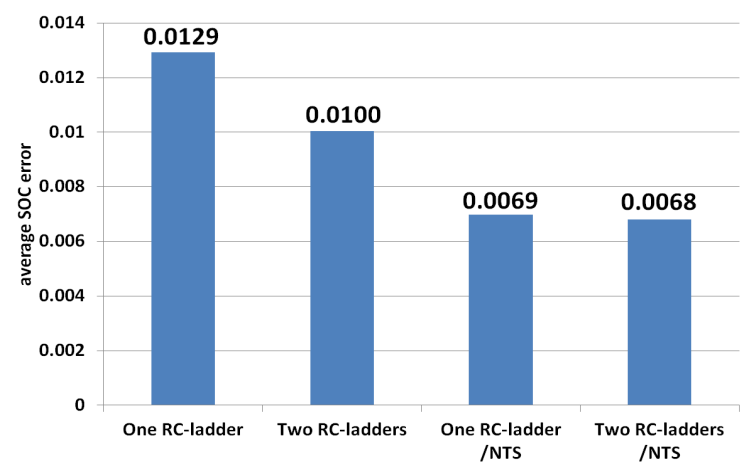

(b)

Fig. 12. Comparison of SOC estimation errors related to the NST of the $\mathrm{LiCoO}_{2}$ cell; (a) Comparison of SOC estimation errors, (b) Comparison of average SOC estimation errors

\subsection{EECM and SOC estimation of the $\mathrm{LiFePO}_{4}$ cell}

\subsubsection{Related to the number of RC-ladder}

Fig. 13 shows the comparison of the SOC estimation with regard to the number of $\mathrm{RC}$-ladder in the $\mathrm{LiFePO}_{4}$ cell. Experimental results clearly show that the SOC estimation accuracy significantly increases when the number of RC-ladder increases. However, in both the cases, SOC estimation error is gradually divergent as shown in Fig. 14. This is because the extraordinary OCV parameter trajectory caused by the hysteresis characteristic

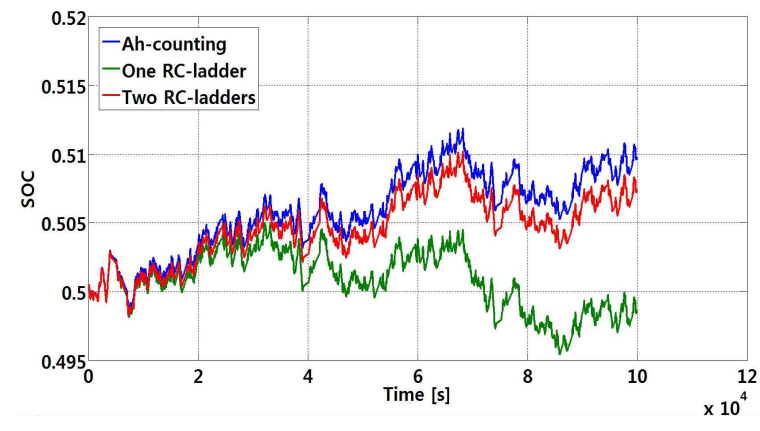

Fig. 13. Comparison of SOC estimation results related to the number of RC-ladder of the $\mathrm{LiFePO}_{4}$ cell

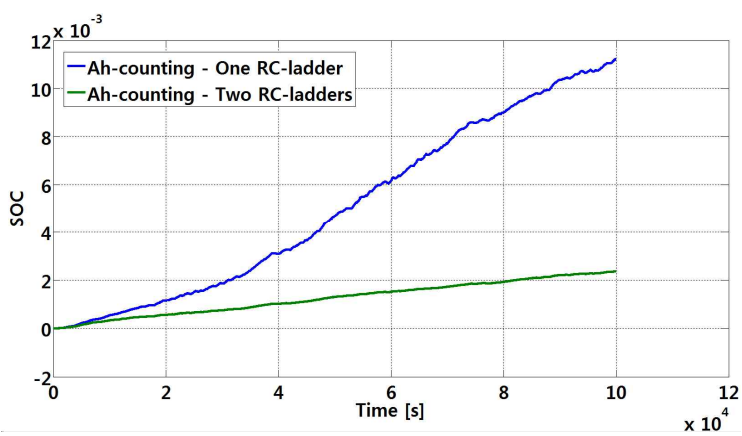

(a)

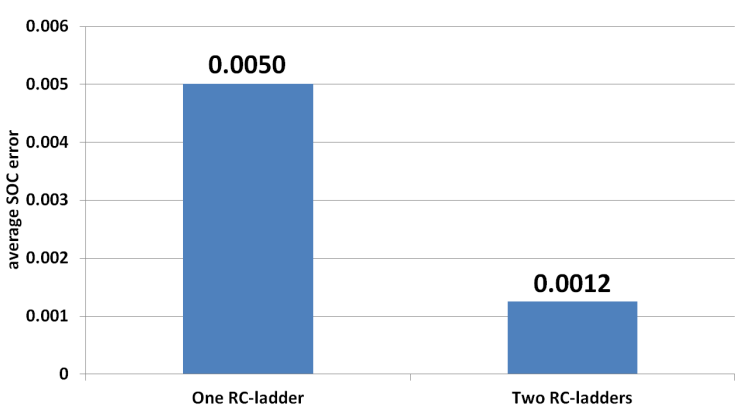

(b)

Fig. 14. Comparison of SOC estimation errors related to the number of the RC-ladder of the $\mathrm{LiFePO}_{4}$ cell; (a) Comparison of SOC estimation errors, (b) Comparison of average SOC estimation errors

of the $\mathrm{LiFePO}_{4}$ cell has not been successfully modeled leading to a gradual accumulation of the model error. An accurate modeling of the OCV is necessary for SOC estimation of the $\mathrm{LiFePO}_{4}$ cell [23].

\subsubsection{Related to the NST}

Under consideration of one and two RC-ladders, Fig. 15 shows the comparison of the SOC estimation related to the NST of the $\mathrm{LiFePO}_{4}$ cell. The accuracy of SOC estimation is enhanced when the NST is applied. However, it can be seen that the SOC estimation error still increases gradually, (see Fig. 16). The effectiveness of the NST is lower than 


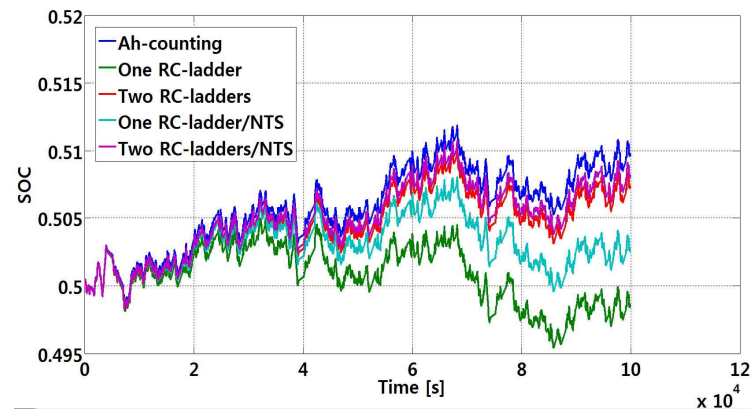

Fig. 15. Comparison of SOC estimation results related to the NST of the $\mathrm{LiFePO}_{4}$ cell

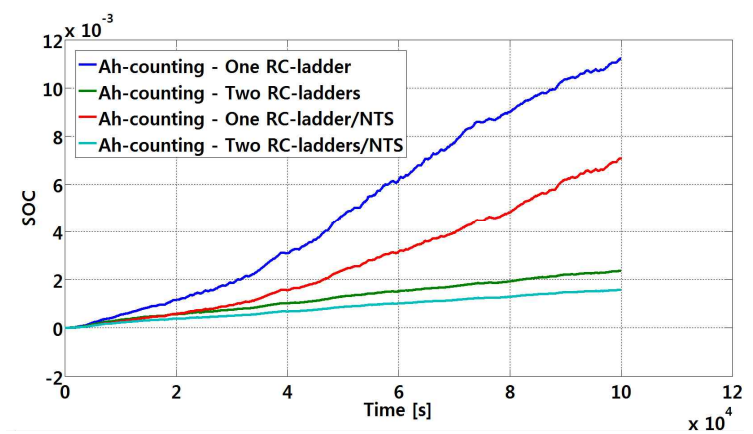

(a)

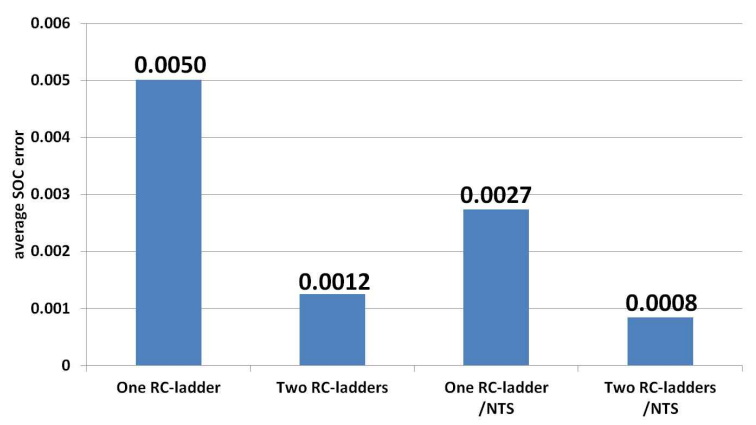

(b)

Fig. 16. Comparison of SOC estimation errors related to the NST of the $\mathrm{LiFePO}_{4}$ cell: (a) Comparison of SOC estimation errors; (b) Comparison of average SOC estimation errors

the number of RC-ladder, different from the case of the $\mathrm{LiCoO}_{2}$ cell. This is because the $\mathrm{LiFePO}_{4}$ cell is relatively strong for external noise as compared with the $\mathrm{LiCoO}_{2}$ cell. Because of the hysteresis phenomenon, the accurate EECM modeling is important to the $\mathrm{LiFePO}_{4}$ cell instead of the NST [23].

\subsubsection{Related to the OCV hysteresis effect}

Fig. 17 shows experimental results of the SOC estimation with respect to the OCV hysteresis effect of the $\mathrm{LiFePO}_{4}$ cell. It can be shown that the accuracy of SOC estimation is greatly improved. In addition, the SOC estimation error is saturated at a certain level, as shown in Fig. 18. In contrast to the major loop in the EECM, the minor loop

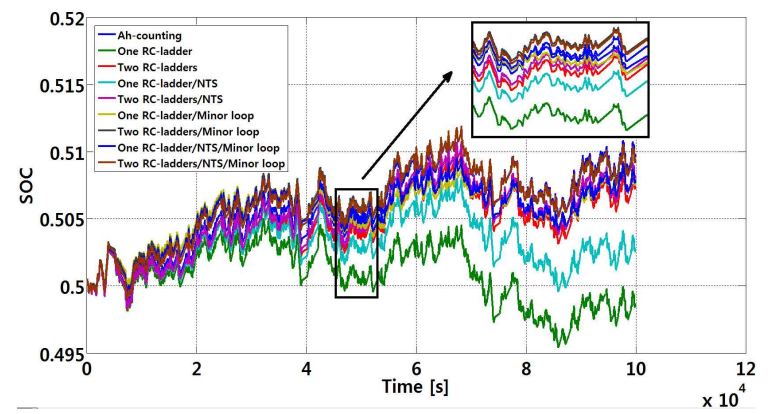

Fig. 17. Comparison of SOC estimation results related to the OCV hysteresis effect of the $\mathrm{LiFePO}_{4}$ cell

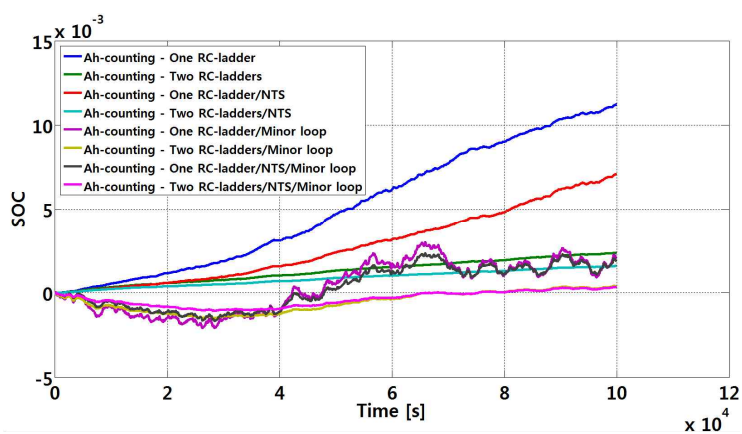

(a)

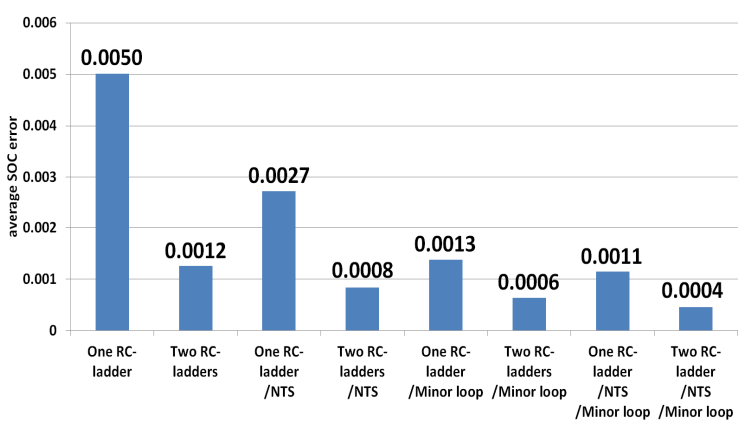

(b)

Fig. 18. Comparison of SOC estimation results related to the OCV hysteresis effect of the $\mathrm{LiFePO}_{4}$ cell:

(a) Comparison of SOC estimation errors; (b) Comparison of average SOC estimation errors

application greatly improves the accuracy of OCV in the EECM, even under the OCV hysteresis effect of the $\mathrm{LiFePO}_{4}$ cell [23].

\subsection{EECM and SOC estimation of the $\mathrm{LiNiMnCoO}_{2}$ cell}

\subsubsection{Related to the number of RC-ladder}

Like two aforementioned cells, the comparative analysis on SOC estimation of the $\mathrm{LiNiMnCoO}_{2}$ related to the number of RC-ladder and the NST is identically implemented. With an adjusted charging/discharging HEV current profile of 20Ah, Fig. 19 displays the comparative analysis of SOC estimation with regard to the number of 


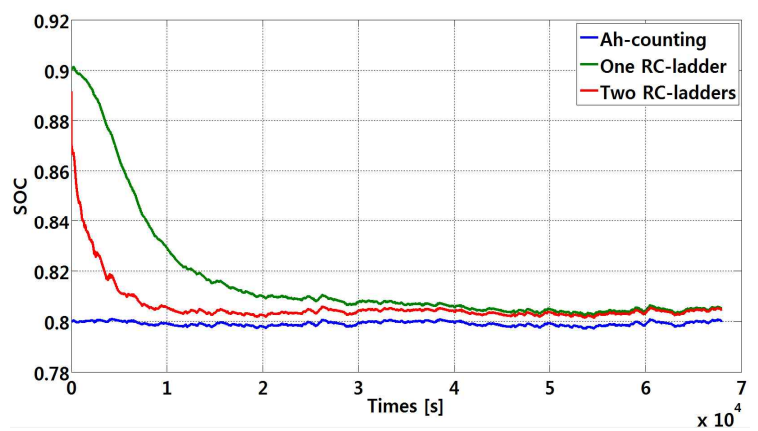

Fig. 19. Comparison of SOC estimation results related to the number of RC-ladder of the $\mathrm{LiNiMnCoO}_{2}$ cell

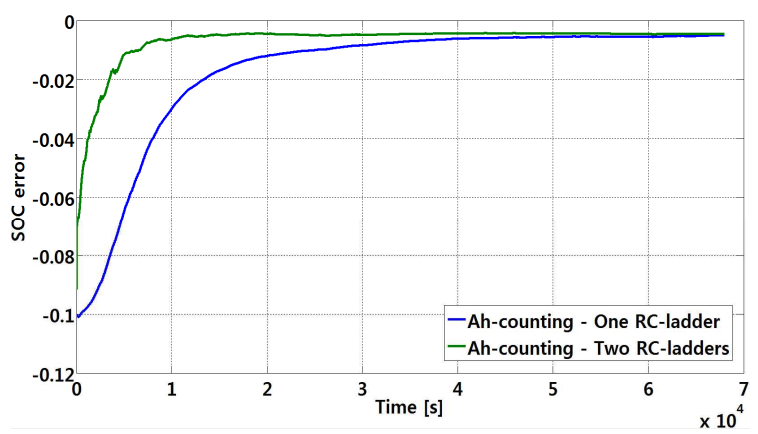

(a)

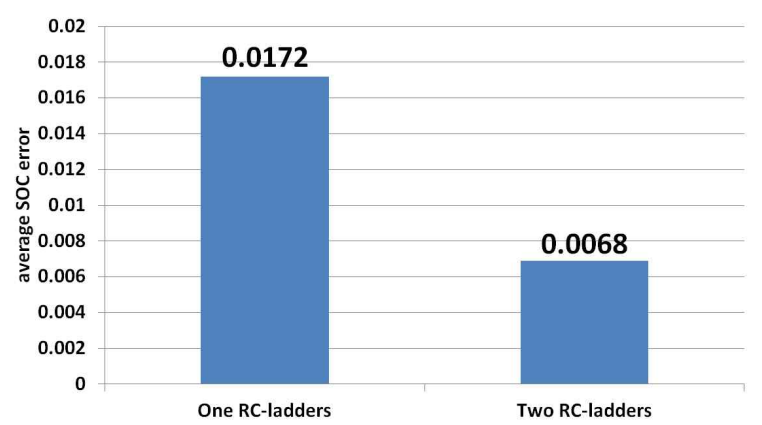

(b)

Fig. 20. Comparison of SOC estimation errors related to the number of the RC-ladder of the $\mathrm{LiNiMnCoO}_{2}$ cell; (a) Comparison of SOC estimation errors, (b) Comparison of average SOC estimation errors

RC-ladder. From Fig. 20, the performance on SOC estimation using two RC-ladders in the EECM is superior to that of one $\mathrm{RC}$-ladder.

\subsubsection{Related to the NST}

In addition to the comparative analysis on SOC estimation using one or two RC-ladders in Section 4.3.1, this section presents the SOC estimation related to the NST of the $\mathrm{LiNiMnCoO}_{2}$ cell in Figs. 21 and 22. These figures clarify that The SOC estimation is clearly dependent on the NST. Moreover, it can be seen that the effect of the NST is greater than that of the number of RC-ladder.

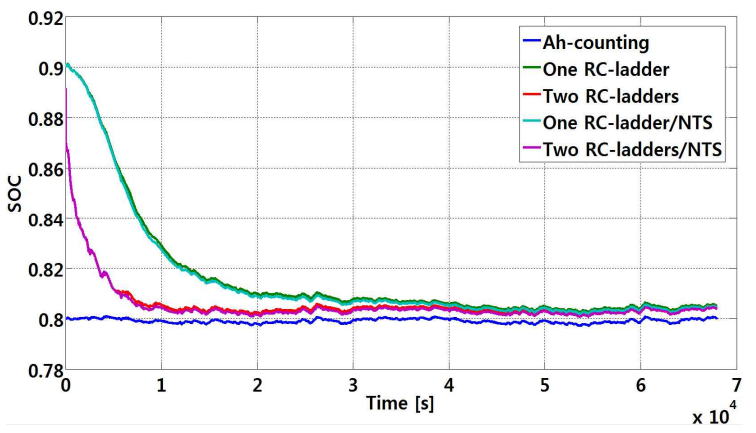

Fig. 21. Comparison of SOC estimation errors related to the NST of the $\mathrm{LiNiMnCoO}_{2}$ cell

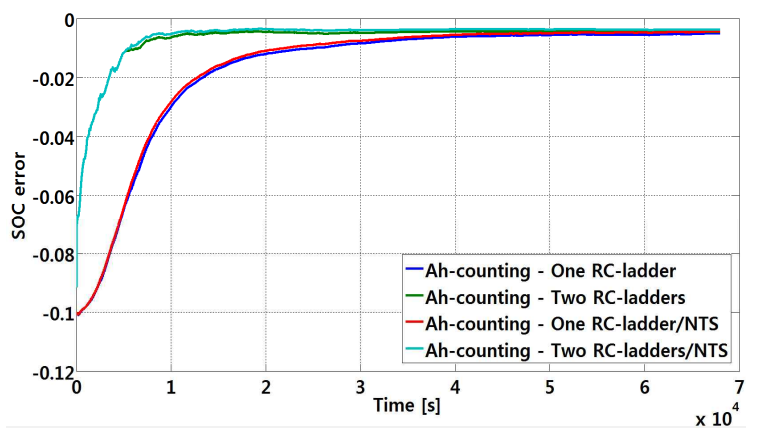

(a)

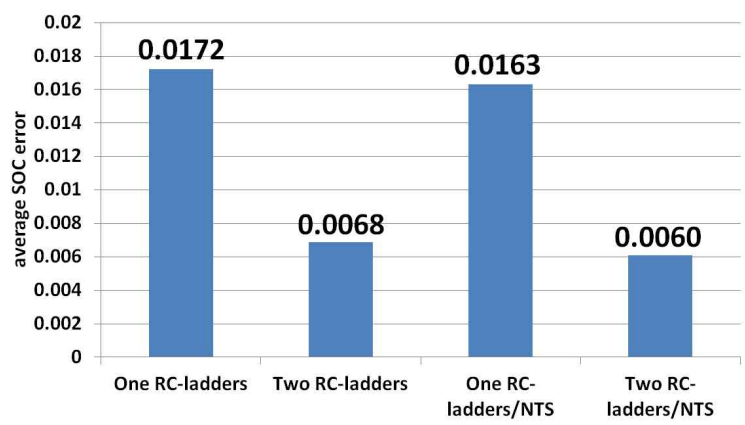

(b)

Fig. 22. Comparison of SOC estimation errors related to the NST of the $\mathrm{LiNiMnCoO}_{2}$ cell; (a) Comparison of SOC estimation errors, (b) Comparison of average SOC estimation errors

\subsection{Discussion of experimental results}

For correct SOC estimation of the $\mathrm{LiCoO}_{2}, \mathrm{LiFePO}_{4}$ and LiNiMnCoO 2 cells, the basic EECM is enough to obtain a certain level of accuracy for the estimation. The use of two RC-ladders is more useful than that of one RC-ladder. But, these cells are weak for external noise, namely EECM error, it is required to use a detector such as NST in the estimation algorithm. This NST includes two measurement noise models and data rejection technique. Each NST was respectively set to three cells. Specifically, in case of the $\mathrm{LiFePO}_{4}$ cell SOC estimation, it is absolutely significant to achieve the exact EECM with well-designed 
OCV modeling by the application considering major loop and minor loop. In this overall perspective, if it is possible to increase number of RC-ladders and the NST technique, a more accurate SOC estimation can be obtained.

\section{Conclusion}

This approach elaborately compares some of the SOC estimation performance results of three representative $\mathrm{LiCoO}_{2}, \mathrm{LiFePO}_{4}$ and $\mathrm{LiNiMnCoO}_{2}$ cells with the EECM and the NST. Consequently it is clearly observed that parameterization, NST, and application of minor loop are very effective for SOC estimation. For $\mathrm{LiCoO}_{2}$ and LiNiMnCoO 2 cells, it is easy to model the static and dynamic characteristics by the EECM; however, due to its sensitivity to external noise, a complement to the algorithm, such as the NST is required. On the other hand, a $\mathrm{LiFePO}_{4}$ cell can resist external noise well. In addition, it has an unusual OCV curve, caused by the hysteresis phenomenon. Thus, the design of minor loop and parametrization is more critical to the SOC estimation than the NST. Evidently, the best way for improving the accuracy of SOC estimation would be to use all the methods. Unfortunately, these methods complicate the algorithm realization. Thus, these methods should be suitably selected and used taking into consideration the characteristics of the battery.

\section{Acknowledgements}

This research was supported by National R\&D Program through the National Research Foundation of Korea (NRF) funded by the Ministry of Science, ICT \& Future Planning (NRF-2016M1A3A1A02021173).

\section{References}

[1] Lee H, Park J, Kim J. SOC estimation performance comparison based on the equivalent circuit model using an EKF in commercial $\mathrm{LiCoO}_{2}$ and $\mathrm{LiFePO}_{4}$ cells. EVS 28.

[2] Katrašnik T. Analytical method to evaluate fuel consumption of hybrid electric vehicles at balanced energy content of the electric storage devices. Appl Energy 2010; 79: 51-64.

[3] Wang L, Cheng Y, Zou J. Battery available power prediction of hybrid electric vehicle based on improved Dynamic Matrix Control algorithms. J Power Sources 2014; 261: 337-347.

[4] Waag W, Fleischer C, Sauer DU. Critical review of the methods for monitoring of lithium-ion batteries in electric and hybrid vehicles. J Power Sources 2014; 258: 321-339.

[5] Miliani EH. Leakage current and commutation losses reduction in electric drives for Hybrid Electric Vehicle. J Power Sources 2014; 255:266-273.

[6] Chen Z, Mi CC, Xiong R, Xu J, You C. Energy management of a power-split plug-in hybrid electric vehicle based on genetic algorithm and quadratic programming. J Power Sources 2014; 248:416-426.

[7] Torres JL, Gonzalez R, Gimenez A, Lopez J. Energy management strategy for plug-in hybrid electric vehicles. A comparative study. Appl Energy 2013; 114:816-824.

[8] Wu X, Cao B, Li X, Xu J, Ren X. Component sizing optimization of plug-in hybrid electric vehicles. Appl Energy 2011; 88:799-804.

[9] Han J, Park Y, Kim D. Optimal adaptation of equivalent factor of equivalent consumption minimization strategy for fuel cell hybrid electric vehicles under active state inequality constraints. J Power Sources 2014; 267:491-502.

[10] Xu L, Li J, Ouyang M, Hua J, Yang G. Multi-mode control strategy for fuel cell electric vehicles regarding fuel economy and durability. Int J Hydrog Energy 2014; 39:2374-2389.

[11] Xu L, Ouyang M, Li J, Yang F, Lu L, Hua J. Optimal sizing of plug-in fuel cell electric vehicles using models of vehicle performance and system cost. Appl Energy 2013; 103:477-487.

[12] Xue L, Jiuchun J, Caiping Z, LeYi W, Linfeng Z. Robustness of SOC Estimation Algorithms for EV Lithium-Ion Batteries against Modeling Errors and Measurement Noise. Mathematical Problems in Engineering 2015; 90:71-94.

[13] Zhang C, Jiang J, Zhang W, Sharkh S. Estimation of state of charge of lithium-ion batteries used in HEV using robust extended Kalman filtering. Energies 2012, 5:1098-1115.

[14] Chen Z, Fu Y, Mi C. State of charge estimation of lithium-ion batteries in electric drive vehicles using extended Kalman filtering. IEEE Transactions on Vehicular Technology 2013 62:1020-1030.

[15] Xiong R, He H, Sun F, Zhao K. Evaluation on state of charge estimation of batteries with adaptive extended kalman filter by experiment approach. IEEE Transactions on Vehicular Techology 2013; 62:108117

[16] Plett G. Extended Kalman filtering for battery management systems of LiPB-based HEV battery packs: Part 3. State andparameter estimation. Journal of Power Sources 2004; 134:277-292.

[17] Plett G. Extended Kalman filtering for battery management systems of LiPB-based HEV battery packs: part 2. Modeling and identification. Journal of Power Sources 2004; 134:262-276.

[18] Plett G. Extended Kalman filtering for battery management systems of LiPB-based HEV battery packs. Part 1. Background. Journal of Power Sources 2004; 134:252-261. 
[19] Nam O, Lee J, Lee J, Kim J, Cho B. Li-ion battery SOC estimation method based on the reduced order extended Kalman filtering. 4th International Energy Conversion Engineering Conference and Exhibit; I-9.

[20] Kim J, Seo G, Chun C, Cho B, Lee S. OCV hysteresis effect-based SOC estimation in extended Kalman filter algorithm for a $\mathrm{LiFePO}_{4} / \mathrm{C}$ cell. IEEE International Electric Vehicle Conference; 1-5.

[21] Zhang H, Chow M. On-line PHEV Battery Hysteresis Effect Dynamics Modeling. IECON 2010 - 36th Annual Conference on IEEE Industrial Electronics Society $2010 ; 1844-1849$.

[22] Lee H, Park J, Kim J. Comparison of SOC estimation using EKF of the $\mathrm{LiFePO}_{4}$ cell according to minor loop in individual SOC range. The Koerean Institute of Power Electronics Annual Conference 2015; 397398.

[23] Lee H, Park J, Kim J. Comparative evaluation of $\mathrm{LiFePO}_{4}$ cell SOC estimation performance with ECM structure and noise model/data rejection in the EKF for transportation application. IEEE Applied Power Electronics Conference and Exposition 2016.

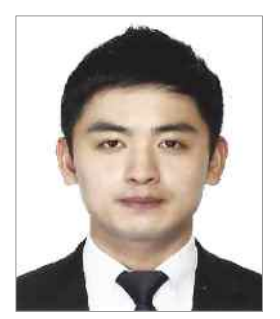

Hyun-jun Lee He received his B.S. degree from the Department of Electrical Engineering of Soongsil University, Seoul, Korea, in 2014. He is currently pursuing his Ph.D. degree at Soongsil University. His current research interests include the equivalent circuit model based SOC performance using the EKF.

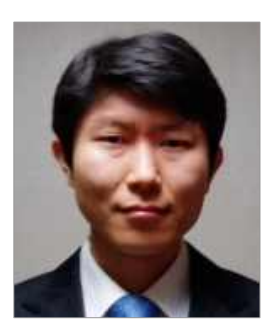

Joung-hu Park He received his B.S., M.S., and Ph.D. degrees from the Department of Electrical Engineering and Computer Science of Seoul National University, Seoul, Korea, in 1999, 2001, and 2006, respectively. He is currently an assistant professor at Soongsil University, Seoul, Korea. His current research interests include the analysis of highfrequency switching converters and renewable energy applications.

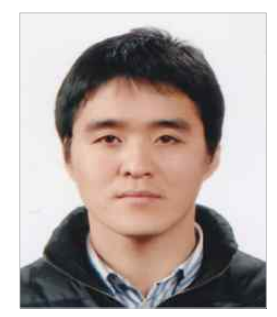

Jonghoon Kim He received the B.S. degree in Electrical Engineering from Chungnam National University, Daejeon, Republic of Korea and Ph.D degrees in Electrical Engineering and Computer Science from Seoul National University, Republic of Korea, in 2005 and 2012, respectively. From 2012 to 2013, he was a Senior Research Engineer in Energy Storage System (ESS) Development Group, Energy Solution (ES) Division, Samsung SDI, Cheonan, Republic of Korea. From 2013 to 2016, he was an assistant professor with the Department of Electrical Engineering, Chosun University, Gwangju, Republic of Korea. He is currently an assistant professor with the Department of Electrical Engineering, Chungnam National University, Daejeon, Republic of Korea. His main research interests include battery management system (BMS; modelling, screening, equalization, $\mathrm{SOC} / \mathrm{SOH}$ estimation), Lithium-air, and fuel cell system (ripple current analysis, fault diagnosis, $\mathrm{SOH}$ prediction, and modelling). He is a member of IEEE and Korean Institute of Power Electronics (KIPE). In addition, he is currently an Associate Editor of the Journal of Power Electronics (JPE). He received the Student Paper Award from IPEC-10. 